\title{
Hypovigilance Detection Based on Eyelids Behavior Study
}

\author{
http://dx.doi.org/10.3991/ijes.v1i1.2927 \\ Belhassen AKROUT and Walid MAHDI \\ Sfax University, Sfax, Tunisia
}

\begin{abstract}
The supervision of people in special circumstances, having as their purpose detecting risk situations, is an area which has many applications. In this context we are interested, in this paper, the problems of driver drowsiness detection based on video analysis. We describe a new approach to detect reduced alertness automatically, to prevent the driver. Unlike many other works, our noninvasively approach is based only on the analysis of geometric features captured form faces video sequence without any electronics elements related to human.
\end{abstract}

Index Terms-Drowsiness Detection, Multi-scale analysis, Head tracking, Circular Hough Transform, Haar Features.

\section{INTRODUCTION}

Drowsiness is the risk of falling asleep for a moment with eyes closed and eyes open at times which is an intermediate state between waking and sleeping. This state is involuntary and is accompanied by reduced alertness. A study of the SNCF [3], the characteristic signs of drowsiness are manifested by behavioral signals such as yawning, decreased reflexes, heavy eyelids, itchy eyes, a desire to close eyes for a moment, a need to stretch, a desire to change positions frequently, phases of"micro sleeps" (about 2-5 seconds), a lack of memory of the last stops and trouble keeping head up.

In literature, many systems based on video analysis have proposed for drowsiness detecting [25] [10] [13] [23] [24]. Special attention is given to the measures related to the speed of eye closure. Indeed, the analysis of the size of the iris that changes its surface according to its state in the video allows the de-termination of the eye closure [6] [11] [13] Other work is based on detecting the distance between the up-per and the lower eyelids in order to locate eye blinks. This distance decreases if the eyes are closed and in-creases when they are open [14] [16] [14] [17].

These so-called single-variable approaches can prevent the driver in case of prolonged eye closure, of its reduced alertness. The duration of eye closure used as an indication varies from one work to another. Sarbjit [11] considers that a person is asleep if the eyes remain closed for a period of 5 to 6 seconds; in this case, we speak of total sleep. On the other side, sleep microphones are detected if the driver goes through a state of sleep for a short time (2-3 seconds). For Horng [15], the driver is considered dozing if he / she close their eyes for 5 consecutive images. Hongbiao [14] estimated that the reduced alertness is determined if the distance between the eyelids is less than $60 \%$ for a period of 6.66 seconds.
Yong [17] divides the state of eye opening into three categories (open, half open, closed). This division allows us to conclude the drowsiness of the driver if the eyes are kept closed more than four consecutive images or eyes move from a state of half open to a closed state for eight successive images. Besides, the percentages of detection of fatigue vary in literature. Yong reached $91.16 \%$ of correct average rate for recognition of the condition of the eyes. As for Horng, he explains that the average accuracy rate for detection of fatigue can reach $88.9 \%$. Wenhui [16] achieved $100 \%$ as a correct detection rate. All the last work calculates the results by a study on subjects varying in number from two to ten individuals (four individuals for Horng [6], five subjects for Hiroshi [4], ten people for Tnkehiro [12] and only two for Yong [17]). The second type of approach is called multi-variable. In this context, the maximum speed reached by the eyelid when the eye is closed (velocity) and the amplitude of blinking calculated from the beginning of blink until the maximum blinking are two indications that have been studied by Murray [8] . The latter shows that the velocity amplitude ratio (A/PCV) is used to prevent the driver one minute in advance. Takuhiro [14] uses an infrared camera and suggests five levels of vigilance namely non-drowsy, slightly drowsy, sleepy, rather sleepy, very sleepy and asleep. Takuhiro proposes several criteria: The percentage of eye opening, the number of blinking, the duration of eye closure, the blinking interval and the surface of eye opening. This work has been tested using a base of ten subjects. A correlation rate that is greater than 0.90 is achieved. Picot presents a synthesis of different sizes as the duration to $50 \%$, the PERCLOS $80 \%$, the frequency of blinking and the velocity amplitude ratio. Picot [9] shows that its criteria are more relevant to the detection of drowsiness. These variables are calculated every second on a sliding window of the length of 20 seconds. They are fused by fuzzy logic to improve the reliability of the decision. This study shows a percentage of $80 \%$ of good detections and $22 \%$ of false alarms. The mono-variable approach enables us to detect the state of drowsiness, but in a very advanced stage. We, hence, focus on studying the characteristics which aim at foretelling the driver, about his fatigue, before she falls asleep, by analyzing the speed of closing his eyes.

In the case of multi-variable approaches, we find that some methods are based on the analysis of the EOG signal. This kind of analysis requires technical cooperation between the hardware and the driver. Moreover, these methods need the use of wide range of parameters, which calls for more data for learning. Nevertheless, video-based approaches, rest on the segmentation of the iris of the eye 
so as to extract the features for the subsequent steps. The segmentation of the iris is calculated from the difference of the images, in the case of using infrared cameras. Still, the drawback of such a method lies in the noise sensitivity of the luminance. In this context, the Hough Circular transform method is used to localize the iris. This method shows sturdiness in the face of the desired shape, an ability to adapt even to images with poor or noisy quality as well as an identification of all directions due to the use of a polar description.

Based on the afore-mentioned remarks, we propose in this paper, an approach that determines the state of drowsiness by analyzing the behavior of the driver's eyes from a video.

\section{PROPOSED APPROACH}

This paper presents an approach for driver drowsiness detection [22] by studying the behavior of conductor eyes in real time by an RGB camera (figure 1). This approach requires a critical step presumed through the automatic face detection, first, and the detection of the box that encompasses both eyes, in the step that follows.

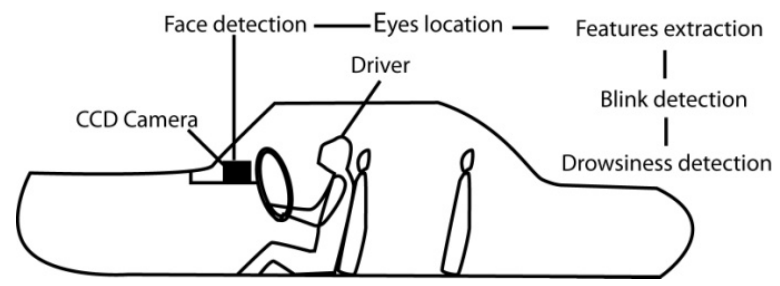

Figure 1. Detection scheme of drowsiness

\section{A. Both Face, eyes localization and tracking}

In order to come to delight the face and the eyes, our approach exploits the object detector of Viola and Jones that is about a learning technique based on Haar features. This method [15] uses three concepts: the rapid extraction of features using an integral image, a classifier based on Adaboost and the implementation of a cascade structure. Figure 2 shows the detection result of the face and the eyes.
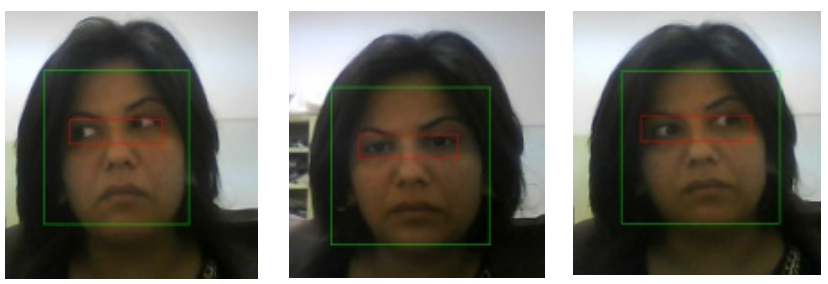

Figure 2. Detection results of both face detection and eye localization

The face and the eyes located by the Haar features are then used to initialize the tracking module. This module is based on the CAMSHIFT [18] algorithm. The choice of this algorithm is argued by its robustness for real-time systems, the invariance to scale and rotation, treatment of occlusions and finally by its insensitivity to object distortions. The CAMSHIFT algorithm steps can be summarized as follows.

\section{CAMSHIFT Algorithm}

1 Select the interest region that contains the object to follow. In our case the face or eyes.

2 Calculate the color histogram of interest region.

3 Calculate the distribution probability of the color histogram.

4 Find the gravity center of the image probability by the Mean-Shift algorithm.

5 Center the search window from the point calculated in step 4 and adjust its size.

6 Return to step 4 until the convergence of the system.

CAMSHIFT algorithm is based on the Mean-Shift algorithm [19]. The Mean-Shift algorithm calculates the center position of interest region. In addition, the CAMSHIFT algorithm calculates the center, the size and orientation of the object. Figure 3 shows some results of detection and face tracking in different positions and lighting conditions.

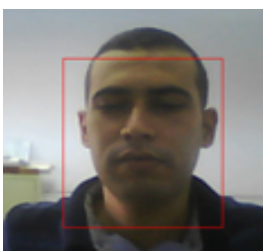

Frame 10

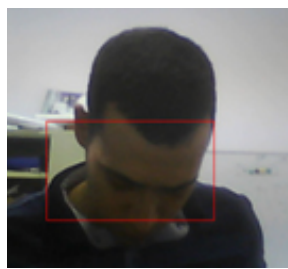

Frame 56

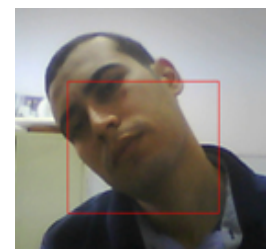

Frame 23

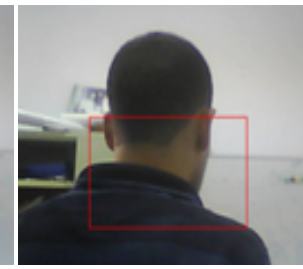

Frame 56

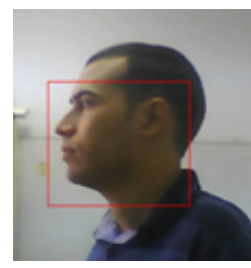

Frame 38

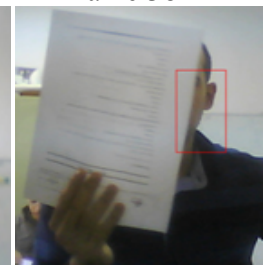

Frame 75
Figure 3. Detection and tracking face results of image sequences using CAMSHIFT algorithm for different conditions

\section{B. Iris and both eyelids Detection}

With reference to the observation of the eye, we note that human eyes are characterized by horizontal contours representing the eyelids and the wrinkles or vertical contours as the ones of the iris. The application of twoscale Haar wavelet allows extracting the vertical, horizontal and diagonal contours. The vertical contours are used in the localization of the iris following application of the Circular Hough Transform. The use of the wavelet allows us to highlight the contours that we want to spot frequently. In our case, the scale of the second rate improves the contours of the iris and the two lids which are going to be detected.

\section{1) Edge extraction based on 2D Haar wavelet}

The Haar wavelet [1] allows us to split the image to find the vertical and horizontal details for the detection of the iris and both eyelids. The wavelet transform is characterized by its multi resolution analysis. It is a very effective tool for noise reduction in digital image. We can keep only the most representative edges. This type of 
analysis is allowed by the multi-resolution. Figure 4 shows the result comparison between the Haar wavelet decomposition and the edge detection with Sobel for the eye image.

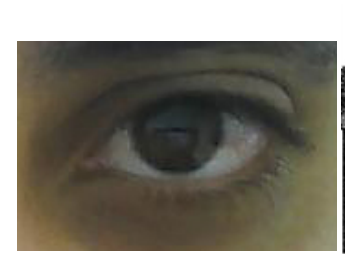

(a)

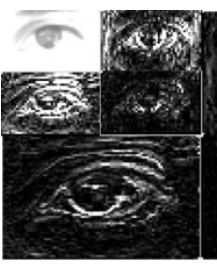

(b) (c)

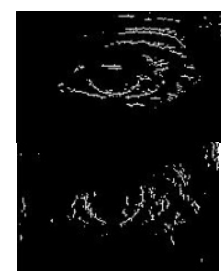

Figure 4. Drowsiness Preprocessing of the eye's image by 2D Haar wavelet decomposition. (a) Original image. (b) Contour detection by Haar wavelet decomposition. (c) Sobel Contour Detection: the horizontal contours on top, the vertical ones at the bottom.

2) Iris and the two eyelids detection based on Circular Hough Transform

Generally, the Hough transform [2] has two spaces. The $\mathrm{XY}$ space and parameter space which varied according to the detected object. The parameter space of the circle is represented by equation 1 .

$$
R^{2}=(x-A)^{2}+(y-B)^{2}
$$

Were $\mathrm{A}$ and $\mathrm{B}$ are the coordinates of the center of the circle and $\mathrm{R}$ is the radius. For reasons of simplification, we can consider that the radius varies between two values in order to reduce the complexity of the algorithm. The equation of the circle can be written in the polar system as follows:

$$
x=r \cos \theta+a \text { and } y=r \sin \theta+b
$$

The process of the research for circles by the application of the Hough transform follows several stages [2]. First, we apply a contour detection algorithm for the decomposition of the Haar wavelet of second rate. For each detected contour pixel, we draw a circle with the desired radius and we suppose that this point is the center of this circle (figure 5).

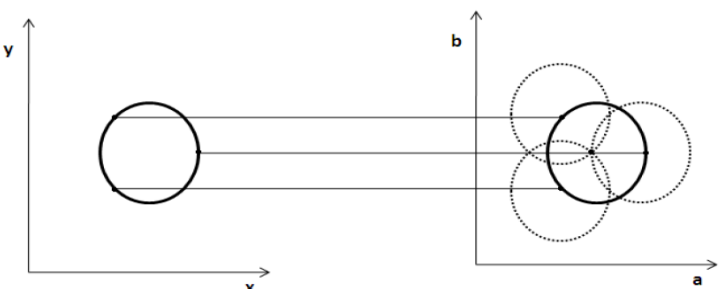

Figure 5. Processed Hough space in the $\mathrm{X}, \mathrm{Y}$ (left) and in the parameter space (right).

This circle is drawn in the space parameter such as the $\mathrm{X}$ axis represents the values of $\mathrm{a}, \mathrm{Y}$ represents the values of $b$ and $Z$ represents the rays. In the case where a point has coordinates belonging to the circle drawn, we raise its position in the accumulator by one. This accumulator has already the same size parameter spaces. Each coordinate $(\mathrm{x}, \mathrm{y})$ of the image becomes a cone (figure 6) in parameter space $(a, b, r)$.

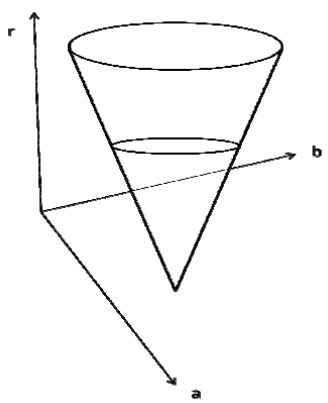

Figure 6. The parameter space used for the circular Hough transform

The maximum value of the accumulator $3 \mathrm{D}$ allows us to determine the coordinates of the center and radius of the circle sought. Our approach involves the detection of the iris by applying the Hough transform on the vertical details of the eye. Both eyelids are located using the Circular Hough Transform on the image of horizontal details of the Haar wavelet decomposition (Figure 7).

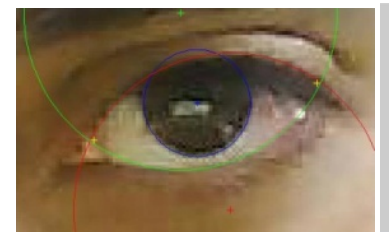

(a)

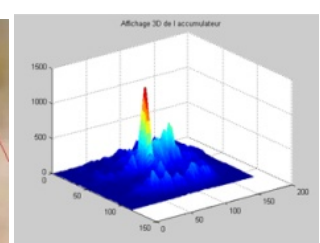

(b)
Figure 7. Both eyelids and iris detected. (a) Result of contour detection by the Circular Hough Transform, (b) 3D shape of the accumulator for the detection of the iris.

\section{Geometric features extraction}

With reference to the detection of the iris, the upper and lower eyelid, we can extract geometric features able to characterize the driver drowsiness state.

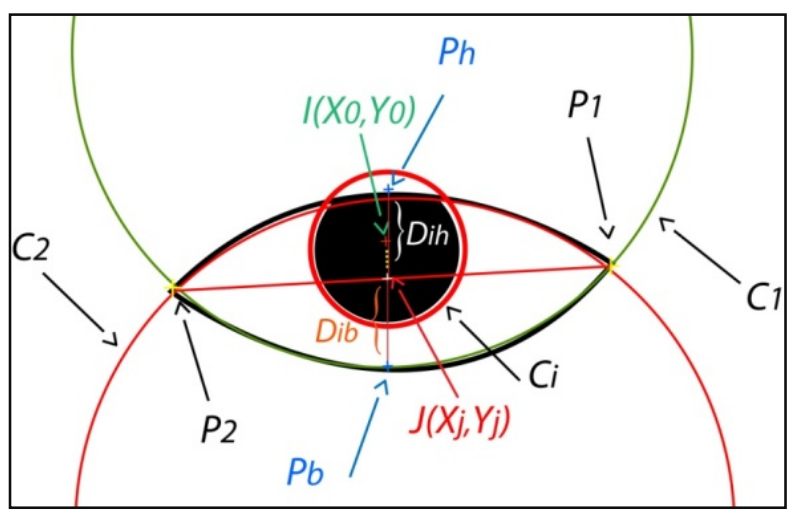

Figure 8. Representation of features from eye figure

We propose two geometric features $D_{i h}$ and $D_{i b}$ (Figure $8)$. These features represent the distance between the point $\mathrm{J}$ and respectively the point $P_{h}$ and $P_{b}$, (Equation 3 ).

$$
\begin{aligned}
& D_{i h}=\sqrt{\left(x_{j}-x_{1}\right)^{2}+\left(y_{j}-y_{1}\right)^{2}} \\
& D_{i b}=\sqrt{\left(x_{j}-x_{2}\right)^{2}+\left(y_{j}-y_{2}\right)^{2}}
\end{aligned}
$$


We note that, $P_{h}$ and $P_{b}$ are calculated from the intersection between the straight line which passes through $\mathrm{J}$ and perpendicular to the line $\left(\mathrm{P}_{1} \mathrm{P}_{2}\right)$ and the two circles $\mathrm{C}_{1}$ and $\mathrm{C}_{2}$, where the equation of one of the two circles is follow as:

$$
R^{2}=\left(x_{0}-x\right)^{2}+\left(y_{0}-y\right)^{2}
$$

And the perpendicular to line $\left(\mathrm{P}_{1} \mathrm{P}_{2}\right)$ is calculated by applying the equation 5 .

$$
y=a x+b=>y^{2}=a x^{2}+b^{2}+2 a x b
$$

By replacing $\mathrm{y}^{2}$ into $\mathrm{R}^{2}$ after development we obtain the equation 6.

$$
\begin{gathered}
(1+a) x^{2}+\left(2 a b-2 x_{0}-2 y_{0}\right) x \\
+\left(b^{2}+x_{0}^{2}+y_{0}^{2}-R^{2}\right)=0
\end{gathered}
$$

The straight line $\left(P_{1} P_{2}\right)$ is determined after the detection of two points $P_{1}$ and $P_{2}$ (Figure 8 ) which represent the intersection of two circles $C_{1}$ and $C_{2}$ such as the squared equation of circle $C_{1}$ is:

$$
R_{1}^{2}=\left(x_{1}-x\right)^{2}+\left(y_{1}-y\right)^{2}
$$

And the squared equation of circle $\mathrm{C}_{2}$ is written as follow:

$$
R_{2}^{2}=\left(x_{2}-x\right)^{2}+\left(y_{2}-y\right)^{2}
$$

The difference between $R_{1}^{2}$ and $R_{2}^{2}$ allows to determinate the tow points $P_{1}$ and $P_{2}$ by the equation 9 .

$$
\begin{gathered}
A x^{2}+x+B\left[x_{1}^{2}+y_{1}^{2}+N^{2}-R_{1}^{2}-2 y_{1} N\right]- \\
R_{2}^{2}+R_{1}^{2}=0
\end{gathered}
$$

Such us:

$$
\begin{gathered}
N=\frac{R_{2}^{2}-R_{1}^{2}-x_{2}^{2}+x_{1}^{2}-y_{2}^{2}+y_{1}^{2}}{2\left(y_{1}-y_{2}\right)} \\
\text { While: } \\
A=\left[\left(\frac{x_{1}-x_{2}}{y_{1}-y_{2}}\right)^{2}+1\right]
\end{gathered}
$$

And:

$$
B=\left[2 y_{1}\left(\frac{x_{1}-x_{2}}{y_{1}-y_{2}}\right)-2 N\left(\frac{x_{1}-x_{2}}{y_{1}-y_{2}}\right)-2 x_{1}\right]
$$

The use of the point $J$ as a reference calculation, not the point $I$ of the center of the iris, allows us to avoid the uncertainties of calculation. These uncertainties are due to the positioning of the point $I$ above the upper eyelid of the eye, resulting in miscalculations of the feature $D_{i h}$ (Figure 9).

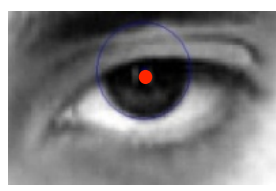

(a)

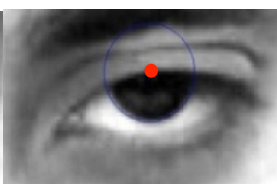

(b)

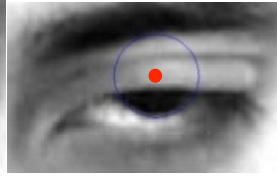

(c)
Figure 9. Different cases of the circle center position of the iris, (a) The center is below the upper eyelid, (b) The center is on the upper eyelid, (c) The center is above the upper eyelid

\section{Experimental study, analysis of eye closure and result of blink detection}

In this section, we describe the experimental studies we conducted to validate the two features $D_{i h}$ and $D_{i b}$ previously proposed. In order to produce realistic data, a human subject is placed in front of our system to simulate different possible movements of the head, the eyelids and the positions of the iris, probably related to different states of fatigue. This experiment consists of studying the temporal variation of both features and normalized the initial state of the eye (Equation 13).

$$
f\left(x_{t}\right)=\frac{D_{i h}^{t}+D_{i b}^{t}}{V_{i}}
$$

The initial value $V_{i}$ is calculated at the beginning of the algorithm when the eyes were open by $75 \%$ (Figure 10 ).

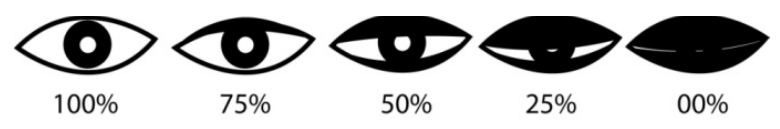

Figure 10. Different states of an open eye

An example of the signal $f\left(x_{t}\right)$ obtained is shown in figure 11 .

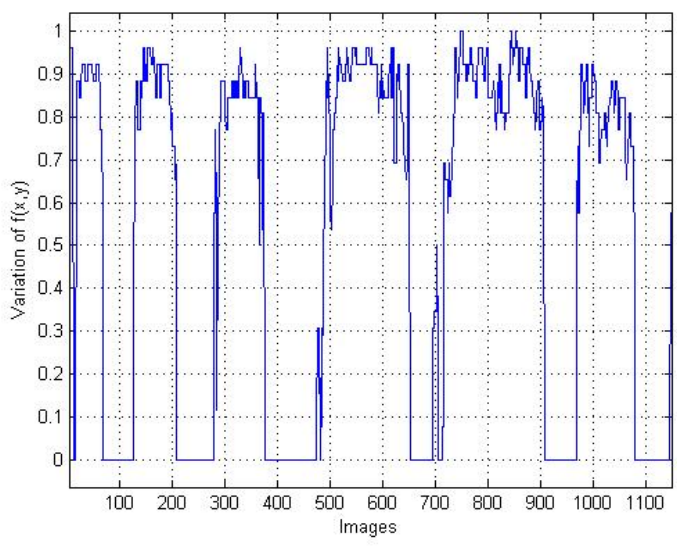

Figure 11. An example of $f\left(x_{t}\right)$ calculated

The first analysis consists of determining the positions state change, whether the beginning eyes closure or the 
beginning of opening. The first derivative of the function $f^{\prime}\left(x_{t}\right)$ allows finding these variations (Figure 13) which are characterized by a negative abrupt change for early closing of the eyes, and by a positive sudden change for early opening of the eye.

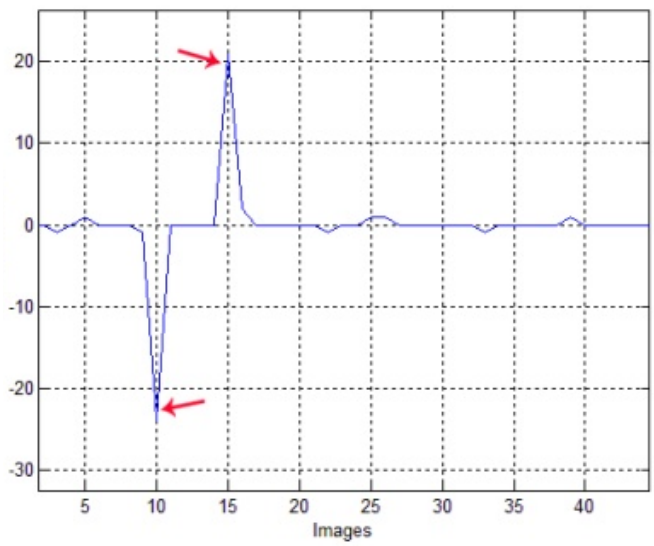

Figure 12. Detection of the blink of an individual by the location of positive and negative sudden change of $\mathrm{f}^{\prime}\left(\mathrm{x}_{\mathrm{t}}\right)$.

Experimentally and after analyzing the video recordings, we found that the derivative of the signal requires a low pass filter to eliminate noise. The selecting of a period considered as blink, is located in the case where $f^{\prime}\left(x_{t}\right)$ falls below (closure) and above (opening) of a well-defined threshold. Sharabaty [20] showed that the maximum duration of a normal blink is 0.5 seconds whereas above this value is considered prolonged closure. In our case the normal or long blink is validated if it satisfies the two previous conditions and its period must exceed 0.15 seconds. Figure 13 shows blink validated with interrupted lines and not validated (between frames 479 and 490) for the signal of $f^{\prime}\left(x_{t}\right)$.

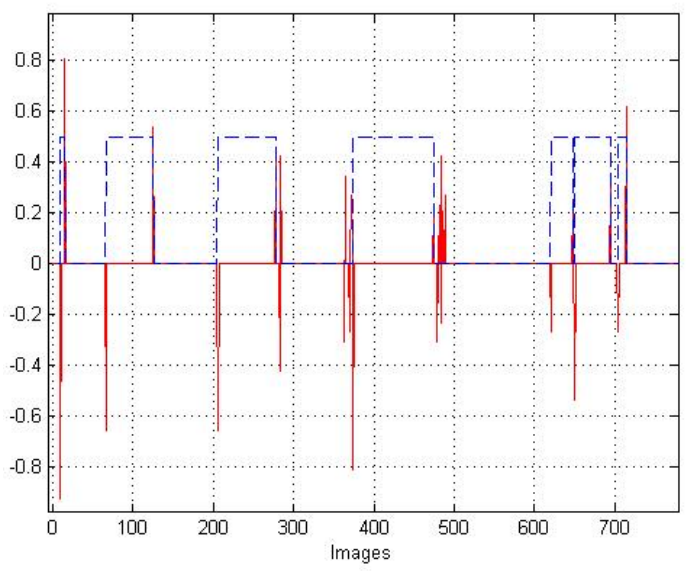

Figure 13. Figure shows the blinking validated

The purpose of the second experiment is to study the difference in closing speed of a person's eyes in a normal state and in another drowsy one. Figure 14 shows the average time of closing of the eyes of a normal person and of a sleepy one. Generally, for a tired individual, the eye closure is slower than that of a vigilant person. This measure can be used as a factor to determinate the level of fatigue.

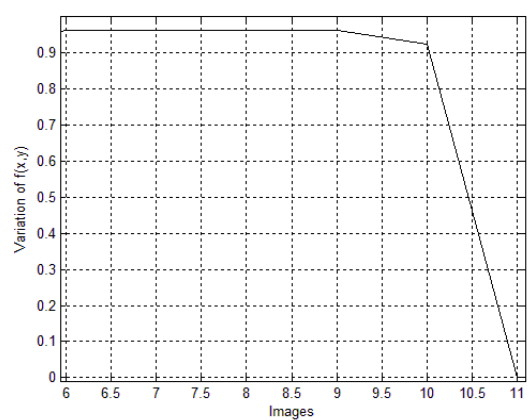

(a)

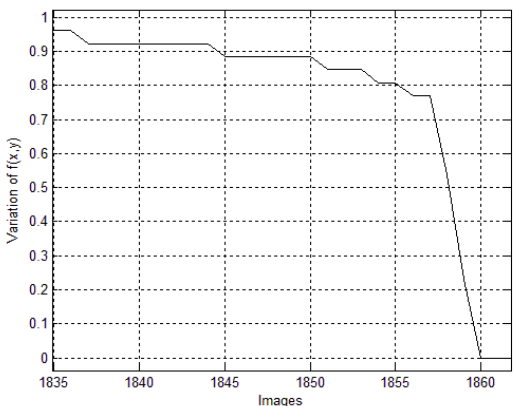

(b)

Figure 14. Speed of eye closure, (a) normal eyes takes $0.16 \mathrm{~s}$, (b) abnormal eyes close in about $1 \mathrm{~s}$

The cumulative change (Equation 14) allows calculation from the period when the individual has their eyes closed.

$$
C\left(x_{t}\right)=\sum_{t=1}^{n} f^{\prime}\left(x_{t}\right)
$$

Fatigue states are characterized by a continuous segment because the evolution of $f^{\prime}\left(x_{t}\right)$ is too low (Figure $15)$.

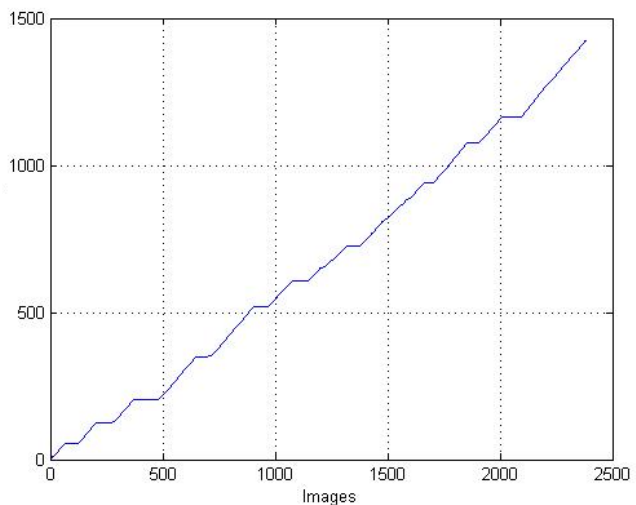

Figure 15. Cumulative curve of $\mathrm{f}^{\prime}\left(\mathrm{x}_{\mathrm{t}}\right)$

This time is lower than 0.5 second [20] for a normal blink and it is greater than two seconds [21] for a blink of an individual in a state of drowsiness (Figure 16). 


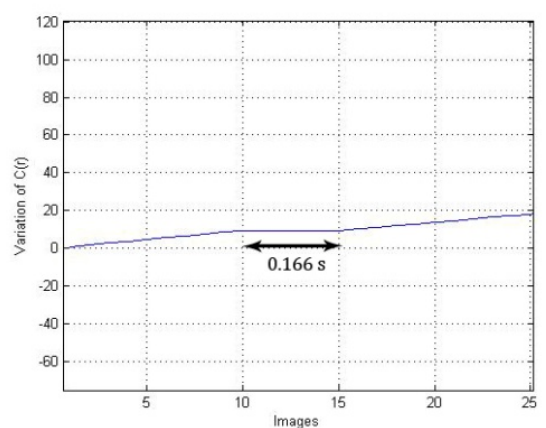

(a)

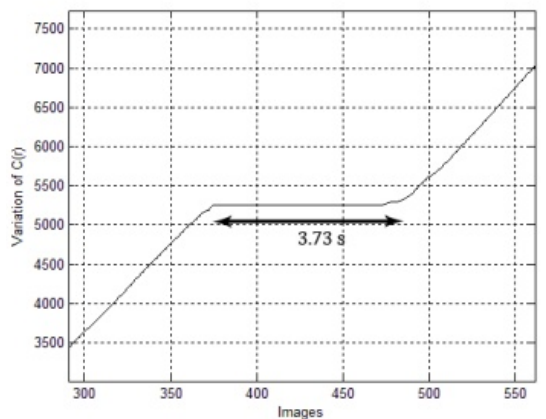

(b)

Figure 16. The period of eye closure, (a) the eyes are closed lower than $0.5 \mathrm{~s}$ for a normal blinking, (b) the eyes in a state of reduced alertness take a period more than $2 \mathrm{~s}$.

In brief, the three conducted experiments show the difference between an alert individual and a sleepy one by analyzing the variation of the features $D_{i h}$ and $D_{i b}$. Generally, the drowsiness states detection is processed by the location of blinking first, then by studying the speed of blinking and finally by calculating the duration of eye closure. The driver is considered in a state of drowsiness if the speed of eye closure exceeds 1 second or the duration of eye closure is greater than 2 seconds. We tested our approach on 10 subjects to determine the detection results of the iris by the Circular Hough Transform (CHT) as shown in Table 1.

TABLE I.

RESUlT OF THE DETECTION OF THE IRIS BY CIRCULAR HOUGH TRANSFORM (CHT)

\begin{tabular}{|c|c|c|c|}
\hline Videos & $\begin{array}{c}\text { Number of } \\
\text { images }\end{array}$ & $\begin{array}{c}\text { False detections of } \\
\text { CHT }\end{array}$ & $\begin{array}{c}\text { Correct rate } \\
\text { detection of CHT }\end{array}$ \\
\hline 1 & 7740 & 246 & $96.82 \%$ \\
\hline 2 & 8280 & 354 & $95.72 \%$ \\
\hline 3 & 7560 & 289 & $96.17 \%$ \\
\hline 4 & 8640 & 394 & $95.43 \%$ \\
\hline 5 & 7920 & 326 & $95.88 \%$ \\
\hline 6 & 7380 & 223 & $96.97 \%$ \\
\hline 7 & 7020 & 186 & $97.35 \%$ \\
\hline 8 & 7386 & 201 & $97.27 \%$ \\
\hline 9 & 8542 & 292 & $96.58 \%$ \\
\hline 10 & 7954 & 252 & $96.83 \%$ \\
\hline Average correct detection by circular Hough & $96.45 \%$ \\
\hline \multicolumn{3}{|c|}{ Transform } \\
\hline
\end{tabular}

The result of the correct average rate is calculated from the average of correct rate of detection (CRT) by circular Hough transform (Equation 15). This value reached $96.45 \%$ as a percentage of detection.

$$
C R T=\frac{\text { Number of images }- \text { False detection }}{\text { Number of images }}
$$

TABLE II.

RESULT OF DROWSINESS DETECTION, FIRST SERIES

\begin{tabular}{|c|c|c|c|c|c|}
\hline Videos & 1 & 2 & 3 & 4 & 5 \\
\hline Real drowsiness & 10 & 12 & 6 & 9 & 9 \\
\hline Generated alarm & 9 & 10 & 6 & 7 & 8 \\
\hline Correct alarm & 7 & 10 & 5 & 6 & 7 \\
\hline Recall & $70 \%$ & $83 \%$ & $83 \%$ & $66 \%$ & $77 \%$ \\
\hline Accuracy & $77 \%$ & $100 \%$ & $83 \%$ & $85 \%$ & $87 \%$ \\
\hline
\end{tabular}

TABLE III.

RESULT OF DROWSINESS DETECTION, SECOND SERIES

\begin{tabular}{|c|c|c|c|c|c|}
\hline Videos & 6 & 7 & 8 & 9 & 10 \\
\hline Real drowsiness & 7 & 4 & 8 & 14 & 5 \\
\hline Generated alarm & 7 & 4 & 8 & 13 & 4 \\
\hline Correct alarm & 6 & 4 & 7 & 12 & 4 \\
\hline Recall & $85 \%$ & $100 \%$ & $87 \%$ & $85 \%$ & $80 \%$ \\
\hline Accuracy & $85 \%$ & $100 \%$ & $87 \%$ & $92 \%$ & $100 \%$ \\
\hline
\end{tabular}

Table 2 and Table 3 show tow series of drowsiness detection result of ten videos test. The opinion of an expert in this step is essential to determine the actual driver drowsiness. The correct rate of fatigue (CRF) is calculated (Equation 16) from the alarms generated by our system and those determined by the specialist.

$$
C R F=\frac{\text { Correct alarm }}{\text { Real drowsiness }}
$$

The average accuracy rate of fatigue which reached $89.6 \%$ is calculated as the average between the accuracy rate of fatigue (ARF) as shown in equation 17 and the correct rate of fatigue.

$$
A R F=\frac{\text { Correct alarm }}{\text { Generated alarm }}
$$

\section{CONCLUSION}

This paper presents an approach to the detection of reduced alertness, based on video analysis. Our system uses a study of the eyes by analyzing the video of several topics.

The steps of detecting drowsiness consist firstly of locating a driver face and eyes by applying Haar features. The circular Hough transform allows the detection of the 
center of the iris and the intersection points of both eyelids (Figure 8) in order to calculate two geometric features. The blink detection, the frequency and the period of eye closure are major factors in determining the individual fatigue.

The guidance of head and other facial feature as yawning can be also an indicator of vigilance state. These data are the subject of our future work in order to improve the obtained results.

\section{REFERENCES}

[1] P. Porwik, A. Lisowska, "The HaarWavelet Transform in Digital Image Processing: Its Status and Achievements", Machine GRAPHICS and VISION. Poland, 2004.

[2] J. Cauchie, V. Fiolet, D. Villers, "Optimization of an Hough transform algorithm for the search of a center", Pattern Recognition. 2008. http://dx.doi.org/10.1016/j.patcog.2007.07.001

[3] T. Guy, P. Baptiste, L. Mohktar, "Expertise CHSCT Unit de production de Brtigny conduite". SNCF. France, 2008.

[4] U. Hiroshi, K. Masayuki, "Development of drowsiness detection system", Vehicle navigation and information systems conference. Japan, 1994.

[5] M. Hongbiao, Y. Zehong, S. Yixu, J. Peifa, "A Fast Method for Monitoring Driver Fatigue Using Monocular Camera", Proceedings of the 11th Joint Conference on Information Sciences. China, 2008.

[6] W. Horng, C. Chen, Y. Chang, "Driver fatigue detection based on eye tracking and dynamic template matching", Proceeding of the 2004 IEEE International Conference on Networking, Sensing and Control. New York, 2004. http://dx.doi.org/10.1109/ICNSC .2004 .1297400

[7] K. Masayuki, O. Hideo, N. Tsutomu, "Adaptability to ambient light changes for drowsy driving detection using image processing", UC Berkeley Transportation Library. 1999.

[8] J. Murray, T. Andrew, C. Robert, "A new method for monitoring the drowsiness of drivers", International Conference on Fatigue Management in Transportation Operations. USA, 2005.

[9] A. Picot, A. Caplier, S. Charbonnier, "Comparison between EOG and high frame rate camera for drowsiness detection", IEEE Workshop on Applications of Computer Vision. USA, 2009.

[10] I. Garcia, S. Bronte, L. M. Bergasa, J. Almazan, J. Yebes, "Vision based drowsiness detector for real driving conditions", IEEE Intelligent Vehicles Symposium. Spain, 2012.

[11] S. Sarbjit, P. Nikolaos, "Monitoring Driver Fatigue Using Facial Analysis Techniques", Intelligent Transportation Systems. Japan, 1999.

[12] O. Takuhiro, N. Fumiya, K. Takashi, "Driver drowsiness detection focused on eyelid behavior", 34th Congress on Science and Technology of Thailand. Thailand, 2008.

[13] M. Tanha, H. Seifoory, "Morphological Drowsy Detection", IEEE International Conference on Signal and Image Processing Applications. Kuala Lumpur, 2011.

[14] I. Tnkehiro, M. Shinji, K. Kduo, N. Tomoaki, Y. Shin, "Driver Blink Measurementby the Motion Picture Processing and its Application to Drowsiness Detection", IEEE International Conference on Intelligent Transportation Systems. Singapore, 2002.

[15] P. Viola, M. Jones, "Rapid object detection using a boosted cascade of simple features", Proc Of CVPR. 2001.

[16] D. Wenhui, W. Xiuojuan, "Driver Fatigue Detection Based On The Distance Of Eyelid", IEEE Workshop Vlsi Design and Video Tech. China, 2005.
[17] D. Yong, M. Peijun, S. Xiaohong, Z. Yingjun, "Driver Fatigue Detection based on Eye State Analysis", Proceedings of the 11th Joint Conference on Information Sciences. China, 2008.

[18] J. G. Allen, R. Y. D. Xu, J. S. Jin, "Object tracking using camshift algorithm and multiple quantized feature spaces", Pan-Sydney Area Workshop on Visual Information Processing VIP2003. Sydney, 2003.

[19] D. Comaniciu, V. Ramesh, P. Meer, "Mean shift: A robust approach towards feature space analysis", IEEE Trans on Pattern Analysis and Machine Intelligence. 2002. http://dx.doi.org/10.1109/34.1000236

[20] H. Sharabaty, B. Jammes, D. Esteve, "EEG Analysis Using HHT: One Step Toward Automatic Drowsiness Scoring”, International Conference on Advanced Information Networking and Applications Workshops. Japan, 2008.

[21] D. Dinges, M. Mallis, G. Maislin, J. Powell, "Evaluation of techniques for ocular measurement as an index of fatigue and the basis for alertness management", Departement of Transportation Highway Safety. USA, 1998.

[22] B. Akrout, W. Mahdi, A. Ben hamadou, "Drowsiness Detection Based on Video analysis Approach", 8th International Conference on Computer Vision Theory and Applications. Spain, 2013.

[23] B. Akrout, W. Mahdi, "Vision based approach for driver drowsiness detection based on 3D head orientation", 7th FTRA International Conference on Multimedia and Ubiquitous Engineering. Springer-verlag. Korea, 2013.

[24] B. Akrout, W. Mahdi, "A blinking measurement method for driver drowsiness detection", 8 International Conference on Computer Recognition Systems Springer-verlag. Poland, 2013.

[25] B. Akrout, W. Mahdi, "A visual based approach for drowsiness detection", IEEE Intelligent Vehicles Symposium. IEEE Intelligent Transportation Systems Society. Australia, 2013.

\section{Authors}

Belhassen AKROUT graduated with a Master's Thesis in Computer Science from the National School of Engineers of Sfax in Tunisia. He is a researcher in the MIRACL laboratory (Multimedia, InfoRmation systems and Advanced Computing Laboratory). Currently, he is preparing his Ph.D, FSEGS- University of Sfax, Tunisia. His research interests include computer vision, video, image and signal processing. He is an IEEE Membership since 2008. (E-mail: akrout_belhassen@yahoo.fr).

Walid MAHDI received his Bachelor degree in business data processing from Sfax University, Tunisia, in 1996 and his Master degree in Data Bases Theory and Engineering from Sorbonne \& Orsay, Paris University, French, in 1997. He received his Doctorate in Informatics from the Central school of Lyon in May 2001. He is actually an Associate Professor in Higher Institute of Computer Science and Multimedia, Sfax-Tunisia. He is also a member of the MIRACL laboratory of Sfax University, Tunisia. His research interests include image and signal processing. He published more than 50 papers in journals and international conferences. $\mathrm{He}$ is a PC member in multiple conferences. (E-mail: walid.mahdi@isimsf.rnu.tn.)

Submitted 19 June 2013. Published as re-submitted by the authors 23 July 2013. 